\title{
Computational Biomechanics for Medical Image Analysis
}

\author{
Adam Wittek, Karol Miller \\ Intelligent Systems for Medicine Laboratory, Department of Mechanical Engineering, \\ The University of Western Australia, \\ 35 Stirling Highway, Perth, WA 6009, Australia
}

\section{INTRODUCTION}

Biomechanics has been traditionally defined as mechanics applied to biology. The quest for understanding the mechanics of living systems has always been regarded as the key subject of biomechanics (Fung, 1993). Consequently, biomechanics has played an important role in understanding the disease and injury mechanisms. The examples include mechanobiology of tumour growth (Chaudhuri et al., 2018), low pressure hydrocephalus formation (Bottan et al., 2012; Dutta-Roy et al., 2008), relationship between loading and femur fracture patterns (Yang et al., 1996), formulation of traumatic injury criteria for the aorta (Belwadi and Yang, 2011), brain (Kikuchi et al., 2015; Margulies and Thibault, 1989; Takhounts et al., 2013; Yang and King, 2011), neck (Bostrom et al., 1996; Matsushita et al., 1997; Ono et al., 1997; Panjabi et al., 2005) and other body parts/organs. The key feature of these studies is that they focus on generic properties and responses of the human body tissues and organs that represent population percentiles and age groups rather than individuals.

At the turn of XXI century it has been envisaged (Bucholz et al., 2004; Graham et al., 2000) that Computer-Integrated Surgery (CIS) systems could extend surgeons' ability to plan and carry out surgical interventions more accurately and with less trauma. CIS systems rely on biomechanical models to estimate complex deformation fields within the body organs. This requires transition from generic understanding of biomechanical phenomena in a human (or animal) organism to patient-specific solutions that address biomechanics of a particular individual. In practice, medical 
images acquired using different modalities are the key source of patient-specific information required for computational biomechanics models representing a particular individual. This includes patient-specific geometry, boundary conditions, loads acting on the body organs and, to limited extent, estimates of the patient-specific material properties. Computational biomechanics models created using such image-derived information can then be applied to predict patient-specific biomechanical responses such as intra-operative deformations for image-guided surgery. Therefore, in this Chapter, we view the roles of biomechanics and medical image analysis in computer assisted intervention as complementary and consider the following two synergistic aspects:

1) Image analysis informs biomechanics - we discuss generation of patientspecific computational models from medical images;

2) Biomechanics informs image analysis - we discuss application of computational biomechanics models in non-rigid image registration. We illustrate the key points of this Chapter with two examples of intra-patient neuroimage registration and an example of whole body computed tomography (CT) image registration.

Soft tissues exhibit non-linear stress-strain relationship (material non-linearity) (Fung, 1993) and undergo large deformations (geometric non-linearity). This is combined with non-linear boundary conditions (through contact interactions of soft tissues with other organs, skull, vertebra, and ribs) and discontinuities induced by surgical dissection. Therefore, while noting the studies that rely on linear elasticity and assume infinitesimally small deformations of the body organs and tissues, we discuss only the solutions and examples that utilise and are compatible with methods of non-linear computational biomechanics that account for geometric and material non-linearity. 
Following main-stream engineering practice, non-linear finite element (FE) analysis (Bathe, 1996; Belytschko et al., 2006) remains a method of choice for computational biomechanics models (Figure 1a). However, the limitations of the FE analysis (for detailed discussion in the context of biomechanics see (Wittek et al., 2011; Wittek et al., 2016), including time consuming generation of computational grids and accuracy and stability deterioration when modelling continua undergoing large deformations, resulted in interest in application of meshless discretisation in biomechanics (Doblare et al., 2005; Li et al., 2016; Miller et al., 2012). Therefore, in our discussion of computational biomechanics in the context of medical image analysis we include both finite element (Figure 1a) and meshless (Figure 1b) methods. The discussion in this Chapter is to large extent based on our previous review of the methods for generating of patient-specific computational biomechanics models (Wittek et al., 2016) and our previous research on computation of brain deformations for image-guided neurosurgery (Joldes et al., 2010; Li et al., 2016; Miller et al., 2012; Miller et al., 2019; Mostayed et al., 2013; Wittek et al., 2007, Zhang et al., 2012).

a)

b)

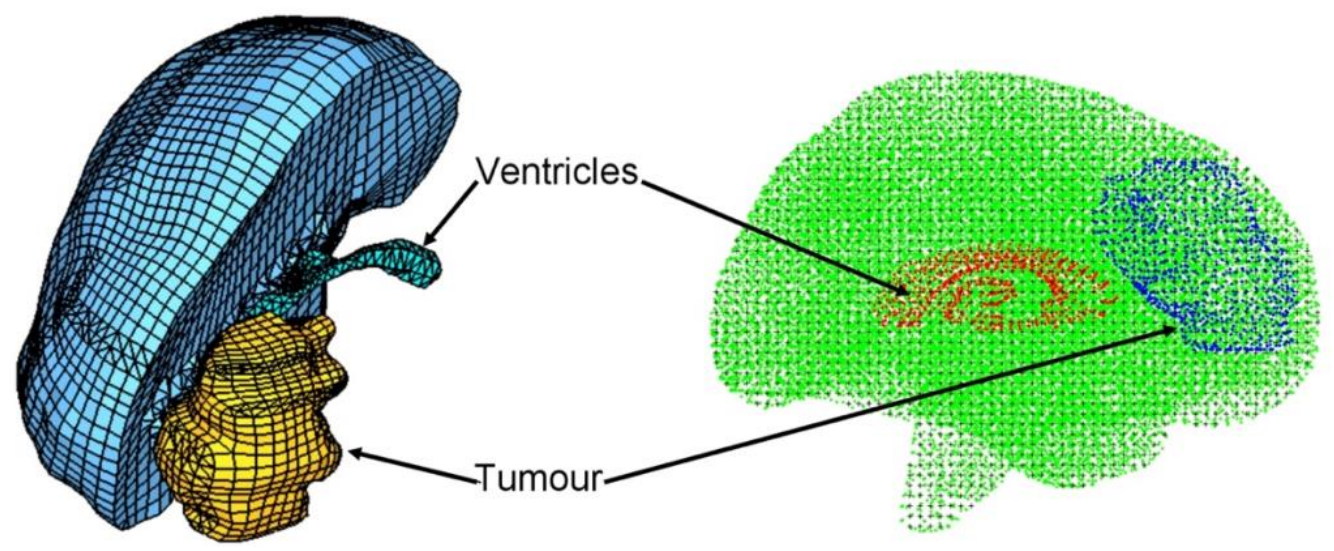

Figure 1 Patient-specific computational models of the brain with tumour. a) Finite element discretisation. Adapted from Wittek et al. (2010). b) Meshless discretisation used in Miller et al. (2012). Adapted from Miller et al. (2012). Blue crosses indicate the integration points, and green circles indicate the support nodes. 


\section{IMAGE ANALYSIS INFORMS BIOMECHANICS: \\ PATIENT-SPECIFIC COMPUTATIONAL BIOMECHANICS \\ MODEL FROM MEDICAL IMAGES 1}

\subsection{Geometry Extraction from Medical Images: Segmentation}

Finite element method utilises a mesh of interconnected hexahedral and/or tetrahedral elements as a computational grid. To generate such grids, enclosed surface that defines the boundary of the analysed continuum (human body organ) is needed (Cavendish et al., 1988; Carey, 1997). Extracting such surface from medical images necessitates segmentation (Figure 2). To render patient-specific FE model generation truly applicable to large clinical studies, segmentation (and other stages of model development) would ideally be automated.

Automated image segmentation remains a challenging task due to the complexity of medical images. Consequently, there is no universal algorithm for segmenting every medical image. The techniques available for segmentation are specific to the application, the imaging modality and anatomic structure under consideration (Wittek et al., 2016). Examples of the techniques that have been used to automate the segmentation of anatomical structures include thresholding (Otsu, 1979), region growing, watershed based methods (Beucher and Meyer, 1993), level set approach (Sethian, 1999), and edge detection algorithms (such as the border detection method (Liow, 1991), Canny edge detection (Canny, 1986), Sobel edge detection and Laplacian edge detection (Davis, 1975).

\footnotetext{
${ }^{1}$ This section is based on Wittek, A., Grosland, N., Joldes, G., Magnotta, V., Miller, K., 2016. "From Finite Element Meshes to Clouds of Points: A review of methods for generation of computational biomechanics models for patient-specific applications". Ann Biomed Eng 44, 3-15.
} 
To improve the segmentation of medical images, anatomical prior information can be used to help delineate anatomical structures. This often involves statistical methods and machine learning approaches. Examples include the Expectation-Maximisation (EM) segmentation algorithm that has been applied in the segmentation of various anatomical structures, such as the brain (Kapur et al., 1996; Pohl et al., 2007) and long bones (femur and tibia) (Ramme et al., 2011).

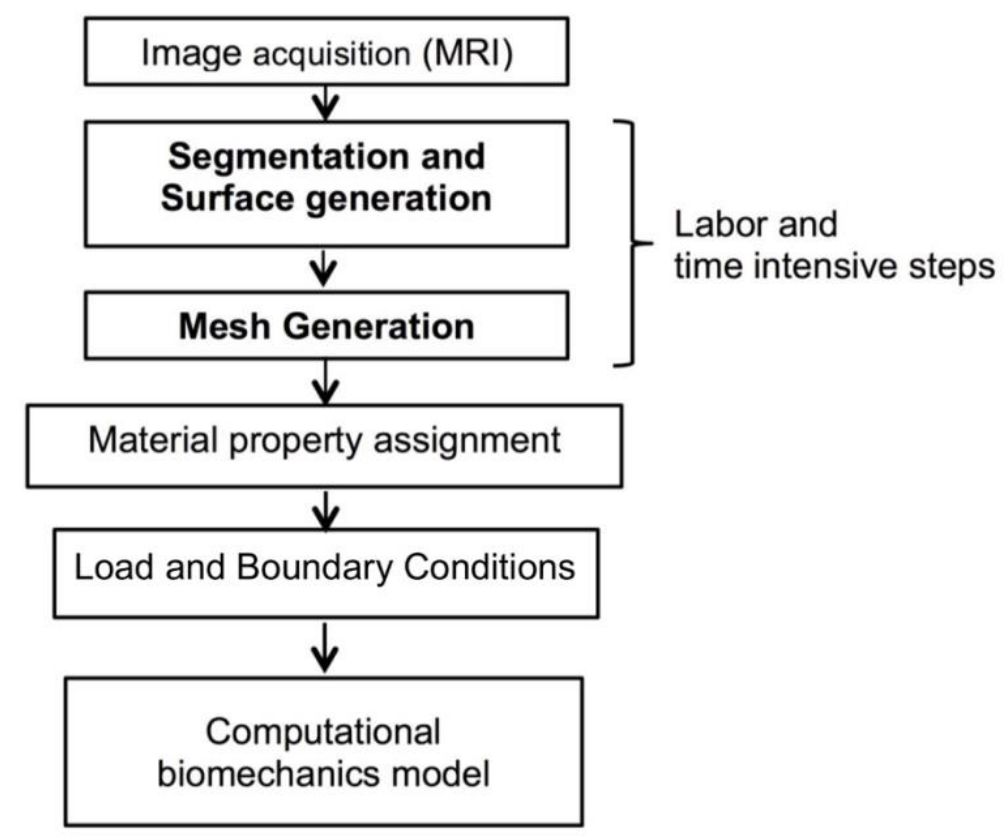

Figure 2 From a medical image to patient-specific computational biomechanics model using finite element method (neurosurgery simulation example). Image segmentation, surface generation and finite element mesh generation often require direct involvement of the analyst and, therefore, are labour intensive. Adapted from Wittek et al. (2016).

\subsection{Finite Element Mesh Generation}

4-noded tetrahedra and 8-noded hexahedra are the most commonly used types of finite elements. Tetrahedral mesh generators are a standard feature of commonly 
used Computer-Aided Engineering CAE packages. They use well-established techniques and optimisation schemes (Choi et al., 2003) including Delaunay triangulation method (Baker, 1989), modified-octree technique (Marechal, 2009), and advancing front technique (Lohner, 1996). Tetrahedral mesh generators available in commercial CAE packages facilitate automated (with the required analyst's input typically limited to the parameters determining the element size, mesh density and element quality) discretisation of objects analysed in engineering applications.

As discussed in section Geometry Extraction from Medical Images: Segmentation when creating patient-specific biomechanical models, information about geometry of the analysed continuum must be extracted (through image segmentation) from medical images. Attempts to address this challenge led to commercial (e.g. Mimics ${ }^{\circledR}$ biomedical.materialise.com/mimics) and open-source (e.g. Slicer3D http://www.slicer.org/) software packages that integrate image segmentation and meshing algorithms. Despite promising results, the quest for automated generation of patient-specific tetrahedral meshes from medical images is far from over as segmentation of images of organs with geometry/anatomy distorted by disease and pathology (such as tumors) still remains a challenge (Wittek et al., 2016) and tends to rely on analyst experience and ability to manually outline boundaries of different anatomical structures in the images. Methods for tetrahedral mesh generation are still an active research topic, as indicated by the regularly held Tetrahedron Workshop on Grid Generation for Numerical Computations (Perotto and Formaggia, 2013; Geuzaine and Renacle, 2016). This includes challenges highlighted in the earlier studies on automated mesh generation, such as variation of element quality according to the mesh generation method employed (Ho-Le, 1988) and element quality control (Shewchuk, 2002; Zhang et al., 2010). 
Although numerous automated generators of tetrahedral meshes are available and in many studies such meshes have been used as a method of choice for patientspecific computational grids for biomechanical models, accuracy and robustness of the results obtained using 4-noded tetrahedral elements should not be taken for granted. This is because such elements exhibit artificial stiffening, known as volumetric locking, when applied in modelling of incompressible or nearly incompressible continua such as soft tissues (Hughes, 2000). In the presence of volumetric locking, the results are mesh dependent. Unfortunately, this fact appears to be often overlooked in a peer reviewed process leading to many papers with results potentially affected by volumetric locking being published. Two types of methods to address volumetric locking have been used: 1) Improved linear tetrahedral elements employing a range of countermeasures to prevent locking; 2) Higher-order and mixedformulation elements. The former includes average nodal pressure (ANP) tetrahedral element (Bonet and Burton, 1998) and it's improvement by Joldes et al. (2009a) that provides much better results for nearly incompressible materials than the standard tetrahedral element with only small increase in the computational cost.

Second-order 10-noded and mixed-formulation (displacement-pressure) tetrahedral elements are readily available in commercial and open-source finite element codes. They are effective in dealing with volumetric locking although they do not eliminate locking completely (Rohan et al., 2014). However, their computation cost is at least four times higher than that of standard linear 4-noded tetrahedral elements. The cost difference is even higher for finite element analysis using explicit time stepping. This might be a limiting factor as many important applications, including image-guided surgery, require models consisting of over hundred thousand elements 
to be solved in-real time (in practice tens of seconds (Grimson et al., 1998)) on commodity hardware.

Under-integrated (using the number of Gauss points lower than that required to accurately conduct spatial integration given the order of the interpolating polynomial in the element shape functions) hexahedral elements do not exhibit volumetric locking (Dassault Systemes Simulia., 2014; Joldes et al., 2009a) and are by far the most efficient when explicit time integration schemes are used (Wittek et al., 2007; Yang and King, 2011). However, despite decades of intense effort, there is no automatic hexahedral meshing algorithms available which would work for complicated shapes routinely encountered when modelling human organs. Hexahedral meshing is particularly challenging when tumour and other pathologies affect the patient's anatomy (Wittek et al., 2010). The manual or semi-manual (Grosland et al., 2009) generation of a three-dimensional hexahedral mesh, although often a highly accurate method, requires significant time and operator effort. Consequently, mesh refinements and convergence studies are rarely reported for this type of mesh.

Various automated hexahedral meshing methods such as plastering (Blacker and Meyers, 1993), whisker weaving (Tautges et al., 1996), and octree-based (Ito et al., 2009; Zhang and Bajaj, 2006; Zhang et al., 2010) techniques have been proposed. Although such methods can produce meshes of high quality, element size control remains a challenge. They require substantial level of technical competency and experience from an analyst constructing patient-specific hexahedral meshes - an important constraint in clinical applications where the users are medical professionals rather than finite element and computer science experts. 


\subsection{Image as a Computational Biomechanics Model: Meshless Discretisation}

Mesh generation constitutes the bulk of the setup time for a problem (Figure 2). This is especially true for anatomical hexahedral finite element mesh development. For example, Ateshian et al. (2015) stated that the process of generating a patient-specific articular contact FE model from CT arthrography (CTA) image data takes over one hundred hours for segmentation and mesh generation. Even when a good quality mesh is created, the FE solution method often fails in the case of large deformations, due to problems such as element inversion. Tissue dissection simulation is also difficult, as small and poorly shaped elements are created during the process of rupture simulation (Vemaganti et al., 2011).

Meshless methods of computational mechanics have been recognised as one possible solution for some of these challenges (Doblare et al., 2005; Horton et al., 2010; Jin et al., 2014; Joldes et al., 2012; Zhang et al., 2014). In meshless methods, the field variable interpolation is constructed over an unstructured "cloud" of nodes scattered within the problem domain and on its boundary with the spatial integration conducted over a grid of background integration cells that do not need to conform to the boundary of the analysed continuum (Horton et al., 2010). Such clouds and grids are incomparably easier to generate than finite element meshes.

To fully utilise the merits of meshless discretisation and open path for automated generation of patient-specific models from medical images, we require an approach that would enable us to distinguish between different tissue types depicted in the image while avoiding segmentation. Such approach has been proposed in the previous studies conducted at The University of Western Australia's Intelligent 
Systems for Medicine Laboratory in which segmentation was replaced by fuzzy tissue classification (Figure 3, right column). In fuzzy tissue classification, surfaces are not generated as the material properties are assigned based on the image intensity directly at the integration points (Figure 3). The process is described in detail in Zhang et al. (2012), Li et al. (2015) and Li et al. (2016). Although fuzzy tissue classification may slightly impede the accuracy of stress computation, the verification conducted in Zhang et al. (2012) showed that the overall accuracy is little affected, with the differences between the results of meshless and finite element computations of the brain deformations of up to only $0.6 \mathrm{~mm}$. As the intraoperative MRI resolution is typically of an order of $1 \mathrm{~mm} \times 1 \mathrm{~mm} \times 2.5 \mathrm{~mm}$, these differences can be regarded as negligible for practical purposes. Most importantly the meshless computational grids with the material properties assigned using fuzzy tissue classification become more robust and the entire patient-specific modelling workflow is simplified and much easier to automate (Figure 3 and Figure 4). 
a)

b)

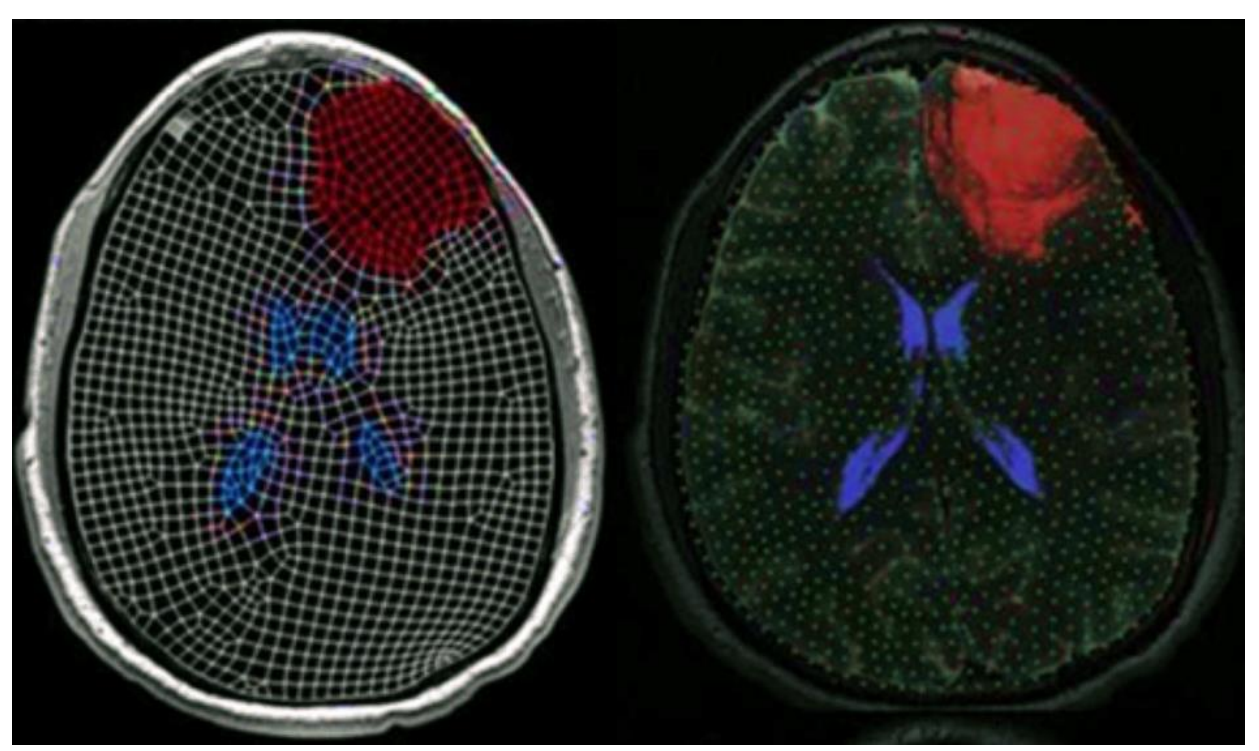

Figure 3 a) Patient-specific finite element model of the brain with the geometry of the parenchyma, tumour (red) and ventricle (blue) obtained through the MRI segmentation. Note clearly defined boundaries between the parenchyma, tumour and ventricles. b) The meshless model of the same brain created using fuzzy tissue classification without the surfaces (boundaries) explicitly separating the parenchyma, tumour and ventricles. The fuzzy membership functions, that describe the probability of a given integration point to belong to the parenchyma, tumour and ventricles, are indicated by the level of red for tumour and blue for ventricles. The support nodes are shown as green circles. Adapted from Zhang et al. (2012). 


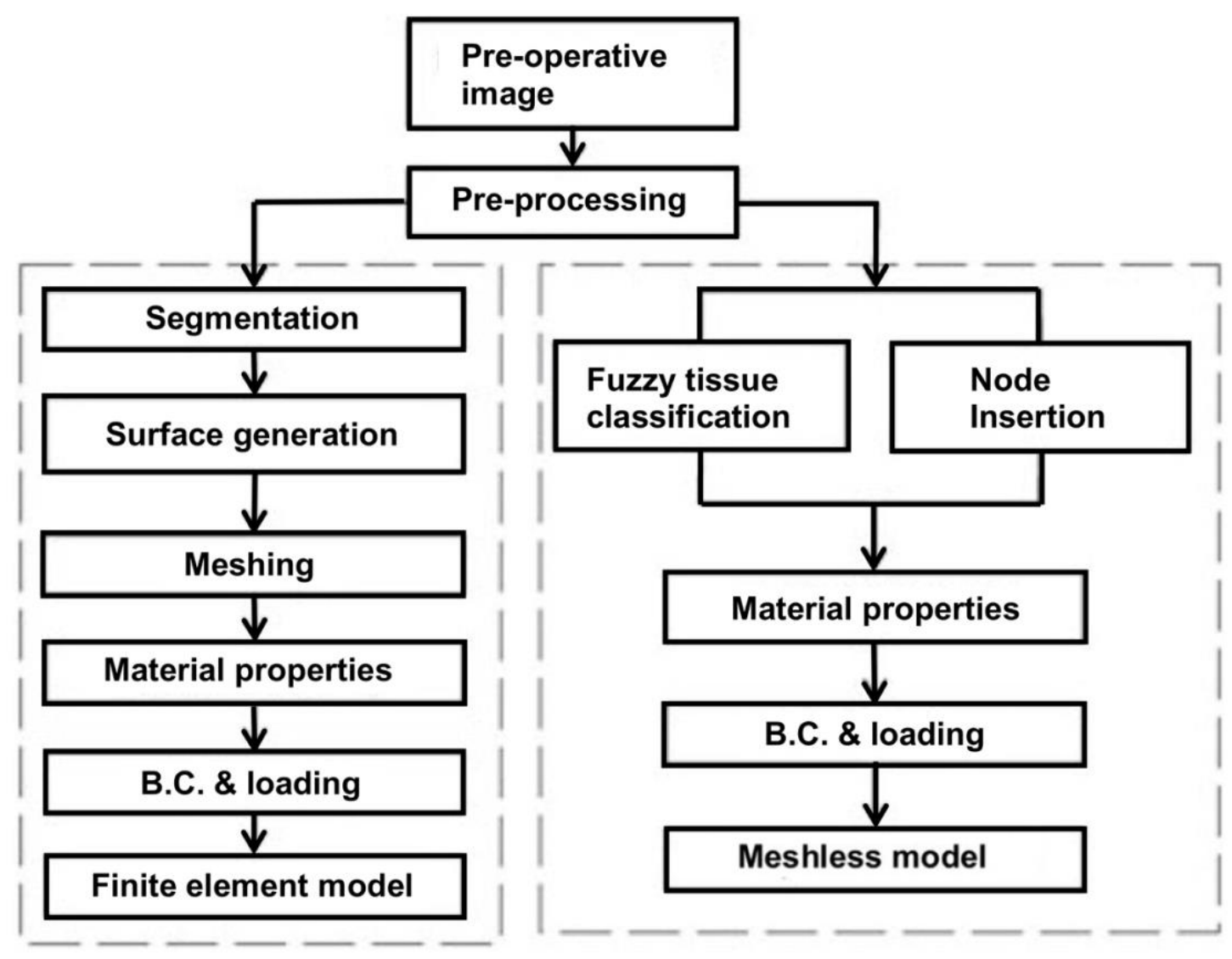

Figure 4 Comparison of the conventional construction of patient-specific computational biomechanics model using image segmentation for defining organ geometry and finite element discretisation (left hand-side column) and the automated process that uses fuzzy tissue classification and meshless discretisation (right handside column). B.C. is the boundary conditions. Adapted from Zhang et al. (2012).

Meshless methods offer a prospect of automated generation of patient-specific computational grids directly from medical images and their robustness in handling of large deformations is superior to the finite element method (Liu, 2003; Li and Liu, 2004; Belytschko et al. 2004). However, important deficiencies of meshless methods, that preclude their use by a non-specialist in the clinical environment, have been highlighted in the literature: 
- Inability to create shape functions for arbitrary grids. Only "admissible node distributions" can be used ( $\mathrm{Li}$ and Liu, 2004). The user must have sufficient knowledge and experience to understand what constitutes an "admissible" grid, and what modifications are necessary if the grid is not "admissible".

- Lack of theoretical error bounds on numerical integration due to the nonpolynomial nature of integrands. Without rigorously established error bounds the solution methods cannot be used in sensitive applications like computational biomechanics for medicine.

Recent developments in meshless algorithms to large extent have eliminated these deficiencies and made meshless methods even more suitable for patient-specific computational biomechanics applications. They include modified moving least squares approximation which can handle almost arbitrary nodal distributions without loss of accuracy (Joldes et al., 2015; Chowdhury et al., 2017), algorithms for efficient and accurate imposition of prescribed displacements on the boundary (a problem referred to in computational mechanics as essential boundary conditions imposition) (Joldes et al., 2017) and adaptive integration that distributes the integration points to achieve the accuracy specified by the user (Joldes et al., 2015). Although these developments do not eliminate the need for general knowledge of computational mechanics when applying meshless methods in creation of patient-specific computational biomechanics models, they make it possible to use such methods as tools without the requirement for expertise in the specific meshless algorithms. 


\section{BIOMECHANICS INFORMS IMAGE ANALYSIS: \\ COMPUTATIONAL BIOMECHANICS MODEL AS IMAGE REGISTRATION TOOL}

In this Section we focus on application of patient-specific computational biomechanics in intra-patient non-rigid registration (that accounts for organ deformations) of medical images. Examples include image-guided surgery, where the high-quality image acquired before the surgery (pre-operative image) needs to be aligned (registered) to lower quality image acquired during surgery (intra-operative image), and diagnosis where the images of the abdominal and other organs acquired for different body posture of the patient need to be compared.

Image registration has been a subject of extensive research effort and numerous publications. Entering a search term "medical+image+registration" in Web of Science database search engine returns over 2,400 journal articles, books and book chapters published in years 1989-2019. Other chapters of this book discuss image registration in great detail. Traditionally image registration has relied solely on image processing methods. We refer to such methods as image-based. The basic components of an image-based registration algorithm are shown in Figure 5. The moving image $\mathrm{M}$ (preoperative image in image-guided surgery) is transformed using the selected transformation $\mathrm{T}$ to obtain the transformed image $\mathrm{T}(\mathrm{M})$. The transformed image is then compared with the fixed image $F$ (intra-operative image in image-guided surgery) using a chosen similarity metric $S$. Then an optimiser is applied to find the parameters of the transform that minimises the difference between the moving and fixed image in the context of the selected similarity metric. The parameters $\Phi$ of the transform are iterated within the optimisation loop to achieve the best agreement between the fixed 
and moving images for the selected metric $\mathrm{S}$. This alone, however, may not ensure plausibility of the predicted deformations as the principles of non-linear biomechanics governing the deformation behaviour of the organ depicted in the image are not taken into account.

The information about the organ deformations/state during surgery acquired using various technologies/modalities is often limited. For instance, the quality of the images acquired during surgery (intra-operative images) may be inferior to that of the images acquired before the surgery (pre-operative images), the intra-operative images may be acquired only for part of the organ or infrequently due to strict time constraints during surgery, geometry of the organ depicted in the image can be strongly affected by surgical dissection, tumour resection, the moving and fixed image may be acquired in different modalities (pre-operative image using MR and intra-operative using CT or ultrasound). These limitations and constraints may pose a challenge for image-based registration methods as determining parameters for the transform that warps the moving image becomes a formidable task if the differences between the images are large and quality of one of the images is poor.

Unlike the image-based registration, biomechanics-based registration methods do not require a fixed (intra-operative) image to update the moving (pre-operative) image, as the transform (deformation field) is computed using a patient-specific computational biomechanics model (see Figure 6). Rather than relying on optimisation as in image-based methods, the registration accuracy depends on predictive power of the model. The information about the geometry of anatomical features of interest are extracted from the moving image to create a computational grid for the model. This is completed by defining the boundary conditions (e.g. contacts) and material properties for the tissues depicted in the image. The loading is typically defined from sparse 
information available in the fixed image (such as deformation of the organ surface due to surgery). A solver is then applied to compute the transform (deformation field) which is then applied to warp the moving image. As soft tissues exhibit non-linear stressstrain relationship (material non-linearity) (Fung, 1993) and the body organs tend to undergo large deformations (geometric non-linearity) combined with discontinuities induced by surgical dissection, solving the governing equations and computing the transform requires application of methods of non-linear computational mechanics in practice, non-linear finite element and meshless solvers (Figure 6). We discussed generating patient-specific computational models for such solvers in the previous section Image Analysis Informs Biomechanics: Patient-Specific Computational Biomechanics Models from Medical Images.

In this Section, we discuss biomechanics-based registration using the examples of neuroimage registration in craniotomy induced brain shift and whole-body computed tomography (CT) registration. We focus on formulation of the problem and robust algorithms for computing the transform (deformation field).

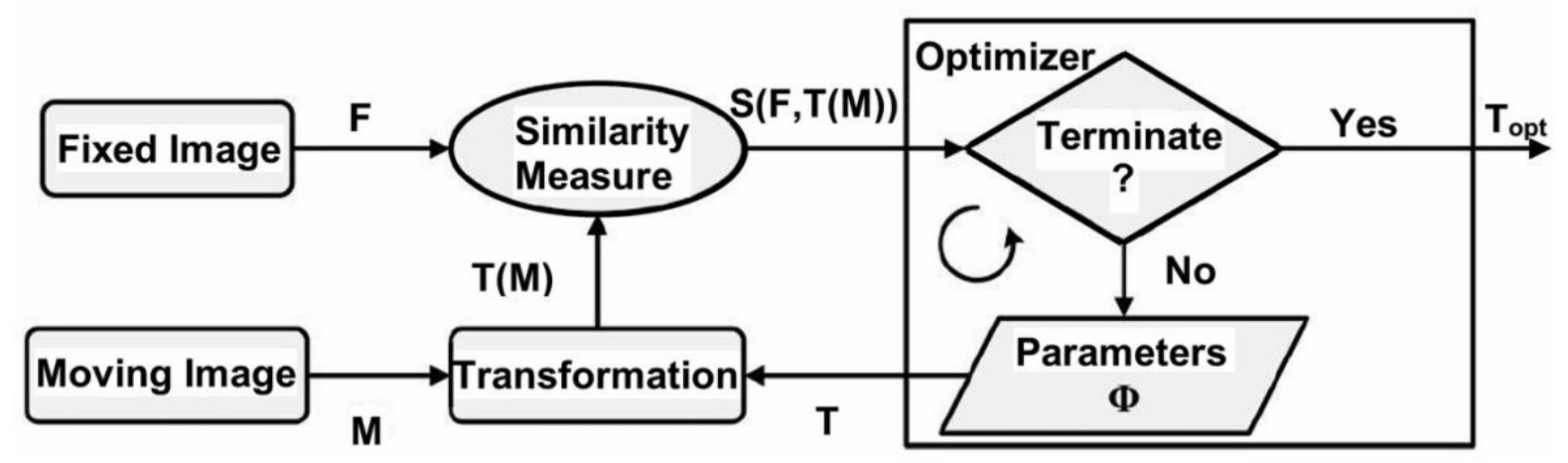

Figure 5 Key components of a general image-based registration process. Modified from Mostayed et al. (2013). 


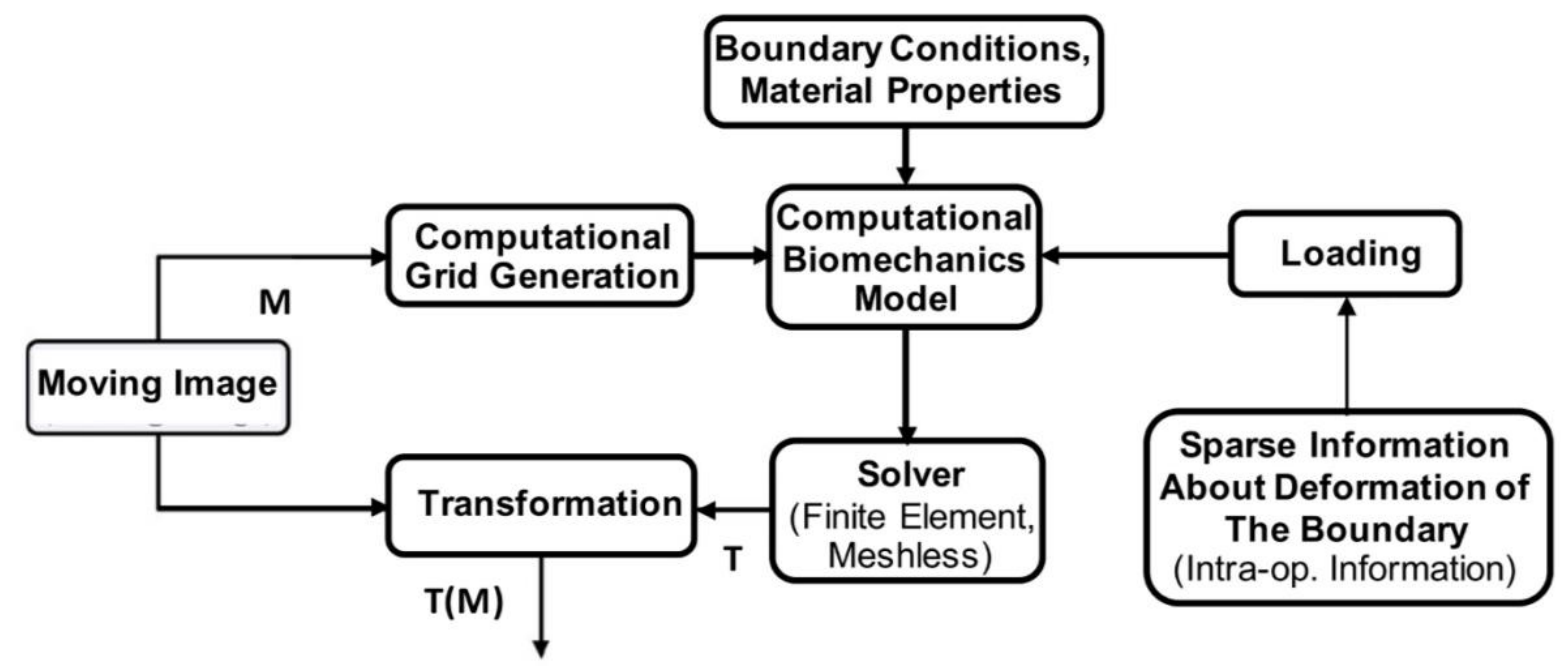

Figure 6 Key components of a general biomechanics-based registration process. In this Chapter we discuss both finite element and meshless solvers. Intra-op. Information indicates the information obtained from intra-operative images (the images acquired during surgery). Modified from Mostayed et al. (2013).

\subsection{Biomechanics-based Image Registration: Problem Formulation}

As explained in Figure 6, only sparse information is required to define the loading that drives the deformation computation in biomechanics-based intra-patient image registration. Loading, boundary conditions and material properties for patient-specific models used in such computations have been discussed in detail in our previous publications (Miller and Lu, 2013; Miller et al., 2011; Wittek et al., 2009; Wittek et al., 2016). Following this previous work, we advocate defining the load through the prescribed displacement of the organ surface and formulating the problem of computing the organ deformations for image registration (in particular for imageguided surgery) in the following way: 
- Known: initial position of the entire domain (as imaged by magnetic resonance MR or computed tomography $\mathrm{CT}$ ) and current position of some parts of the boundary, e.g. the displacement of the exposed (due to craniotomy) surface of the brain (see Figure 6). No surface tractions are applied;

- Unknown: deformation field within the domain (the brain), in particular, current position of the tumour, other pathologies and critical healthy areas.

Problems of this type are referred to as Dirichlet-type problems (Miller and Lu, 2013) or problems with Dirichlet boundary conditions (Ciarlet, 1988). We showed that for Dirichlet-type problems, the predicted deformations are only very weakly sensitive to the variation in mechanical properties of the analysed continua (Wittek et al., 2009). This facilitates accurate patient-specific computational biomechanics modelling without patient-specific information about the tissue mechanical properties. Despite an on-going progress and research effort (for most up-to-date review see Bilston, L. E., 2018), that includes ultrasound (Jiang et al., 2015; Ertl et al., 2018), magnetic resonance (MR) (Guo et al., 2013; Hiscox et al., 2016) and optical elastography, there is no reliable noninvasive method to determine constitutive properties of human soft tissues in-vivo. Nevertheless, our previous results (Garlapati et al., 2014; Li et al., 2015; Li et al., 2016; Mostayed et al., 2013) discussed in the next Section as well as the results by other research groups (Bucki et al., 2010; Fan et al., 2017) indicate that for medical image registration formulated as a Dirichlet-type problem of computational mechanics, clinically relevant results can be obtained even without the knowledge of patient-specific material properties of soft tissues. 


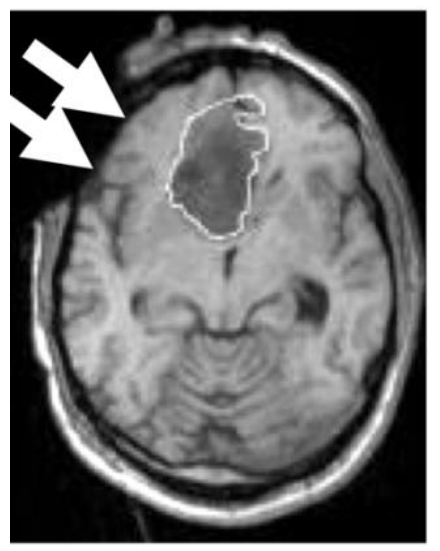

Figure 7 Schematic representation of the load defined by prescribing the displacements (white arrow lines) on the organ surface (in the craniotomy area in this figure).

\subsection{Biomechanics-based Image Registration: Examples}

\subsubsection{Neuroimage registration 2}

As the brain undergoes deformation (known as brain shift) following the craniotomy, intra-operative localisation of the tumour boundaries is a well-recognised challenge in neurosurgery. Although such localisation in principle can be achieved using intraoperative imaging, acquiring intra-operative MRI is often cumbersome and intraoperative MR scanners are expensive. Hardware limitations of these scanners make it infeasible to achieve frequent whole brain imaging during surgery. An alternative approach is to acquire rapid sparse intra-operative data and use it to predict the deformation for the whole brain. An example is the study by Mostayed et al. (2013) in which intra-patient registration of high-quality MRI to intra-operative brain geometry was performed using biomechanics-based and image-based (BSpline interpolation implementation in 3DSlicer, www.slicer.org, open source software platform for medical

\footnotetext{
2 This section is based on Mostayed, A., Garlapati, R.R., Joldes, G.R., Wittek, A., Roy, A., Kikinis, R., Warfield, S.K., Miller, K., 2013. "Biomechanical model as a registration tool for image-guided neurosurgery: Evaluation against BSpline registration". Ann Biomed Eng 41, 2409-2425.
} 
image informatics, image processing and three-dimensional visualisation (Fedorov et al., 2012)) methods for 13 retrospective image-set of patients undergoing glioma resection.

In Mostayed et al. (2013), the biomechanics-based registration was performed using fully non-linear (that accounts for both geometric and material non-linearity) total-Lagrangian explicit dynamics finite element algorithm previously developed and verified by our research group for computation of soft continua deformations (Joldes et al., 2009b; Miller et al., 2007). The algorithm was also implemented on a Graphics Processing Unit (GPU) for real-time computations (Joldes et al., 2010), so that the solution of the entire model takes less than $10 \mathrm{~s}$. The patient-specific finite element computational grids (finite element meshes) were created through the image segmentation (Figure 8). Following the formulation of image registration as a Dirichlettype problem of computational mechanics, the loading was defined by prescribing the displacement in on the brain surface in the craniotomy area. Despite using this very sparse intra-operative information and without patient-specific knowledge of the material properties (Young's modulus of $3000 \mathrm{~Pa}$ was used for the parenchyma), the registration accuracy was at least as high as that using BSpline algorithm that used the information from the entire intra-operative image (Figure 9 and 10).

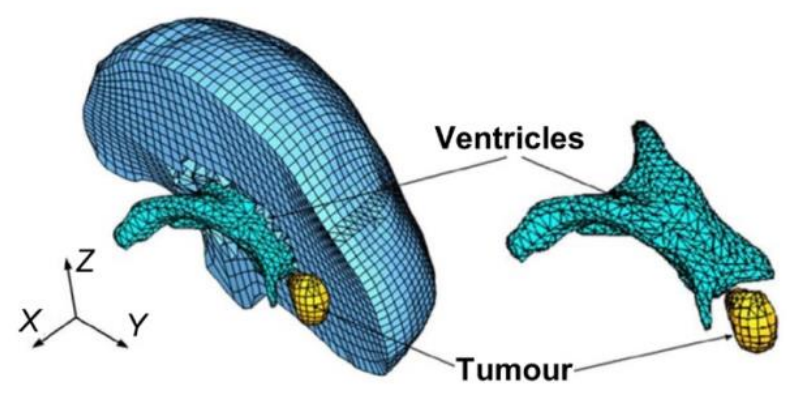

Figure 8 Example of the patient-specific finite element mesh created from the segmented pre-operative MRI used by Mostayed et al. (2013). Adapted from Mostayed et al. (2013). 


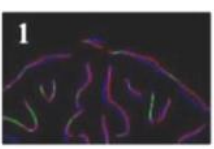

2
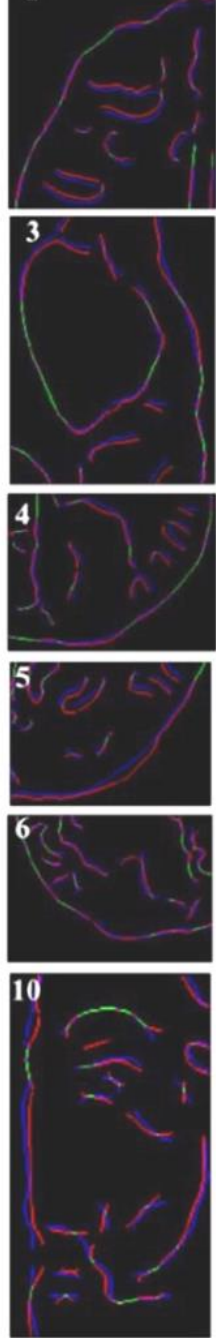

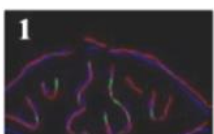

2

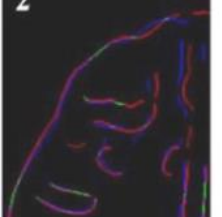

3

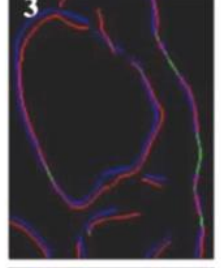

4
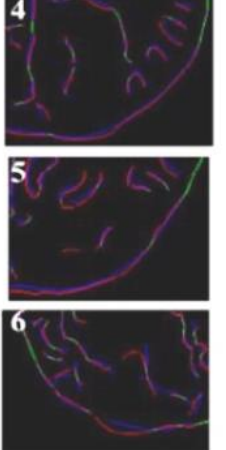

10

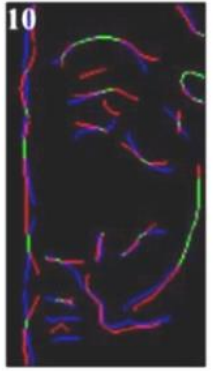

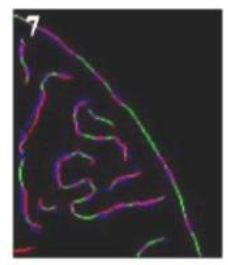
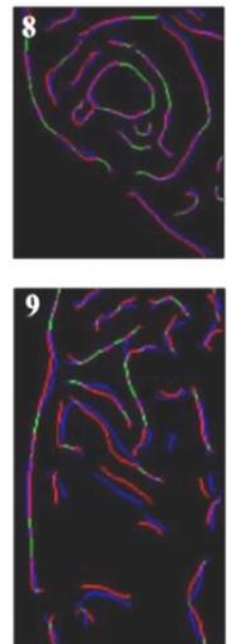

11
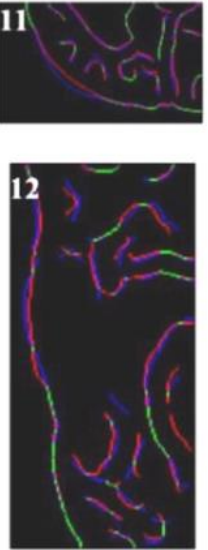
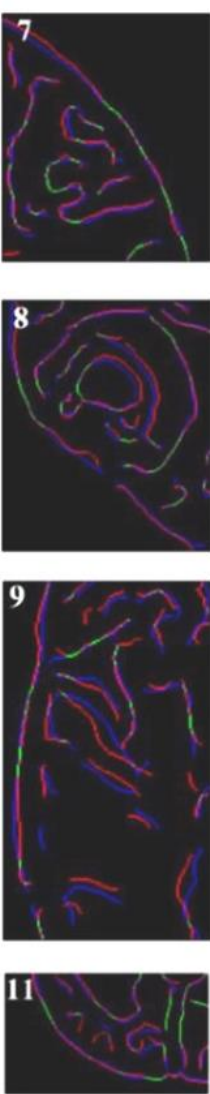

12

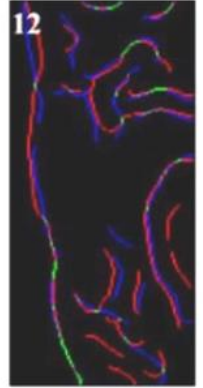

Figure 9 Results for 12 image-sets from Mostayed et al. (2013): qualitative comparison of the registration results obtained using computational biomechanics model (non-linear finite element analysis) and BSpline algorithm. The figure shows the Canny edges (detected using Canny edge filter (Canny, 1986)) in the intra-operative and registered images. Red colour indicates the non-overlapping pixels of the intraoperative slice and blue colour indicates the non-overlapping pixels of the preoperative slice. Green colour indicates the overlapping pixels. The number in each image denotes a particular neurosurgery patient. For each patient (image-set), the lefthand-side image shows edges for the biomechanics-based warping and the righthand-side image shows edges for the BSpline-based registration. Adapted from Mostayed et al. (2013). 

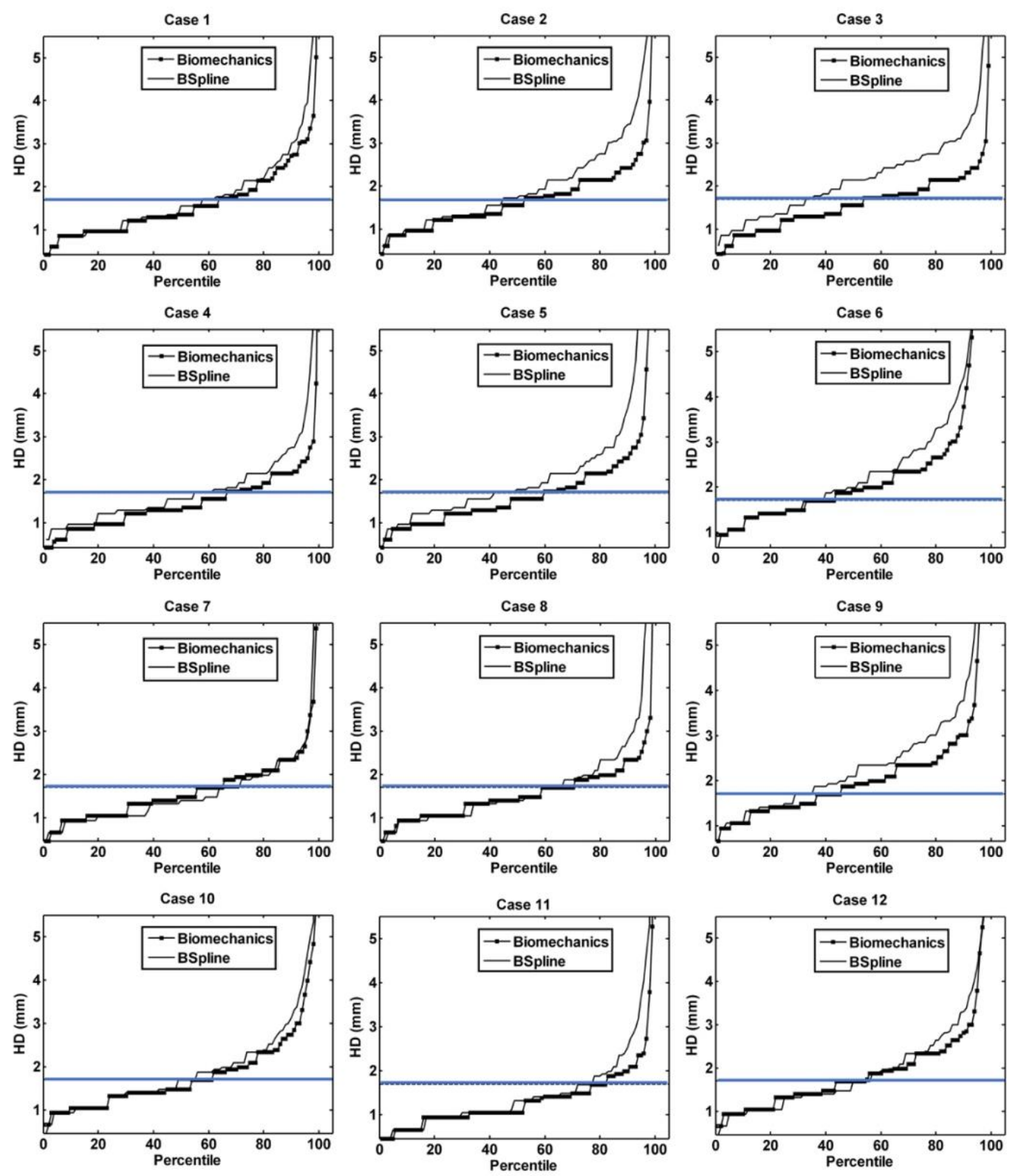

Figure 10 Results for 12 image-sets (cases) from Mostayed et al. (2013): quantitative comparison of the registration results obtained using computational biomechanics model (non-linear finite element analysis) and BSpline algorithm. Each plot shows the Hausdorff distance (Hausdorff, 1957) between the intra-operative and registered preoperative images against the percentile of Canny edges (detected using Canny edge filter (Canny, 1986)) for axial slices. The horizontal line is the $1.7 \mathrm{~mm}$ mark which is the two-times in-plane resolution of the intra-operative images used by Mostayed et al., (2013). Note that the accuracy of biomechanics-based registration is at least as high as that of the image-based (BSpline) registration. For detailed discussion of application of the Hausdorff distance as an image similarity metric, see Garlapati el. (2015). Adapted from Mostayed et al. (2013). 


\subsubsection{Magnetic Resonance (MR) image registration for intracranial electrode localisation for epilepsy treatment 3}

Intracranial EEG (iEEG) is the clinical gold standard for functional localisation of the seizure onset zones (SOZ); invasive electrodes are implanted and monitored for several days, and then removed during a second surgery when the resection is performed (Bauman et al., 2008) (Figure 11). Placement of electrodes must be verified to enable clinicians to associate measured data with pre-operative images of cortical structures. Electrode grids in the intracranial space, and the body's inflammatory response to the craniotomy, displace and deform the brain from the configuration observed in pre-surgical MR imaging. This deformation (brain shift) must be accounted for to ensure accurate alignment of electrode placements and correct clinical evaluation of invasive data. This challenging problem has been addressed in the previous studies conducted at the Intelligent Systems for Medicine Laboratory, The University of Western Australia (Menage, 2017; Psanoudakis, 2017; Miller et al., 2019) using the biomechanics-based registration process summarised in Figure 6. These studies employed patient-specific computational biomechanics models to compute the deformation field within the brain and then apply the computed deformations to register (warp) the pre-operative MRI to the intra-operative (after implanting the iEEG electrodes) brain geometry (Miller et al., 2019; Menage, 2017) (Figure 12). The patient-specific finite element mesh was constructed from the high resolution pre-

\footnotetext{
${ }^{3}$ This section is based on Miller, K., Joldes, G.R., Bourantas, G.C., Warfield, S.K., Hyde, D.E., Kikinis, R., Wittek, A., 2019, "Biomechanical modeling and computer simulation of the brain during neurosurgery" https://arxiv.org/abs/1904.01192 and includes the results from 1) Menage, L.P.M., 2017, "Computer Simulation of Brain Deformations for the Surgical Treatment of Paediatric Epilepsy", MSc Thesis, School of Mechanical and Chemical Engineering, The University of Western Australia, Perth, Western Australia, Australia, and 2) Psanoudakis, N., 2017, "Computer Simulation of Brain Deformation for Paediatric Epilepsy", MSc Thesis, School of Mechanical and Chemical Engineering, The University of Western Australia, Perth, Western Australia, Australia.
} 
operative MRI. The load was defined as imposed displacements (determined from the intra-operative CT).

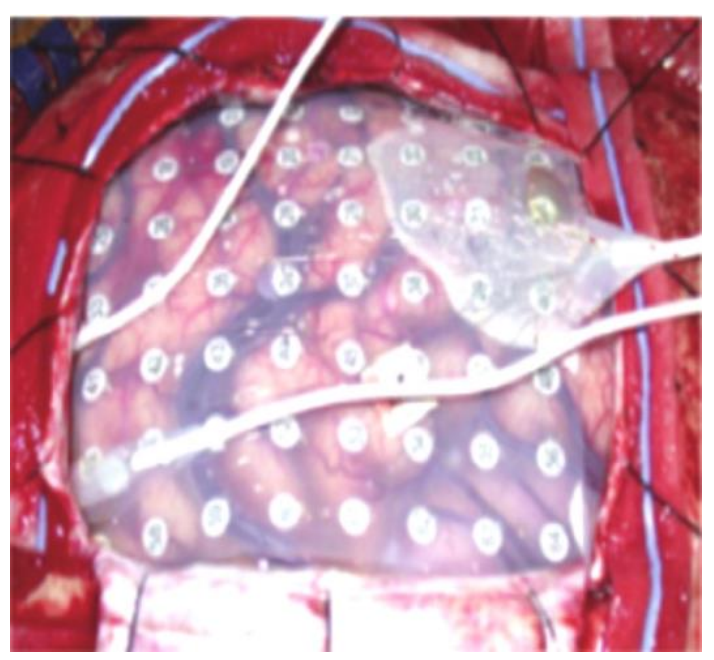

Figure 11 Brain cortical surface exposed by the craniotomy with intracranial EEG (iEEG) implanted. Adapted from Menage (2017) and Miller et al. (2019). The photograph received from Computational Radiology Laboratory (CRL) at Boston Children's Hospital and Harvard Medical School.

a)

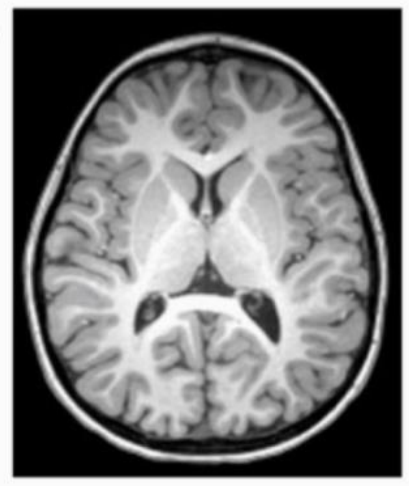

b)

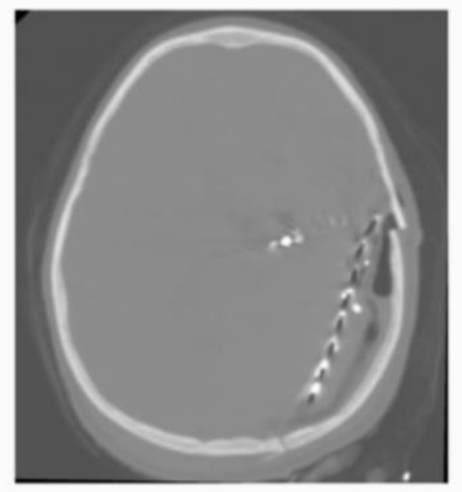

c)

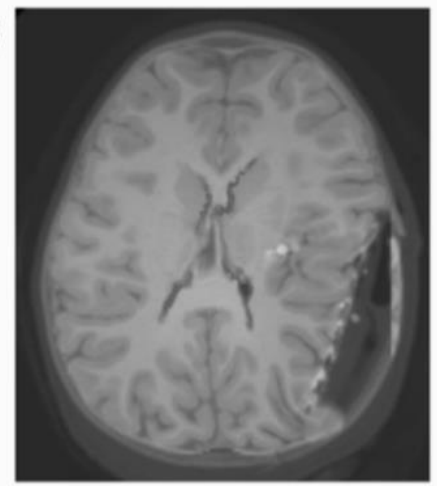

Figure 12 a) Brain pre-operative MRI; b) Intra-operative CT with intracranial EEG (iEEG) electrodes implanted. The electrodes, that define the deformed brain surface, are clearly visible (white points); c) Pre-operative MRI registered onto the intraoperative CT. The figure shows $2 \mathrm{D}$ sections of $3 \mathrm{D}$ image volumes. Adapted from Menage (2017) and Miller et al. (2019). 


\subsubsection{Whole body Computed Tomography (CT) image registration 4}

The study using biomechanics-based whole-body CT registration conducted by Li et al. (2016) is an example of application of fuzzy-tissue classification and meshless discretisation to construct patient-specific computational biomechanics models in a semi-automated fashion (Figure 13). The previously verified Meshless Total Lagrangian Explicit Dynamics algorithm (Horton et al., 2010) was used for solving the governing equations of continuum mechanics. The loading was defined by prescribing the displacements of the vertebrae extracted from the moving and fixed images. Despite using this very sparse information to define the load for computational biomechanics models and distinguishing only eight tissue types when constructing the models (Figure 13b), the results by Li et al. (2016) indicate successful registration of most image features (Figure 14 and Figure 15).

\footnotetext{
${ }^{4}$ This section is based on Li, M., Miller, K., Joldes, G.R., Kikinis, R., Wittek, A., 2016. "Biomechanical model for computing deformations for whole-body image registration: A meshless approach". International Journal for Numerical Methods in Biomedical Engineering 32, e02771-02771-02718.
} 


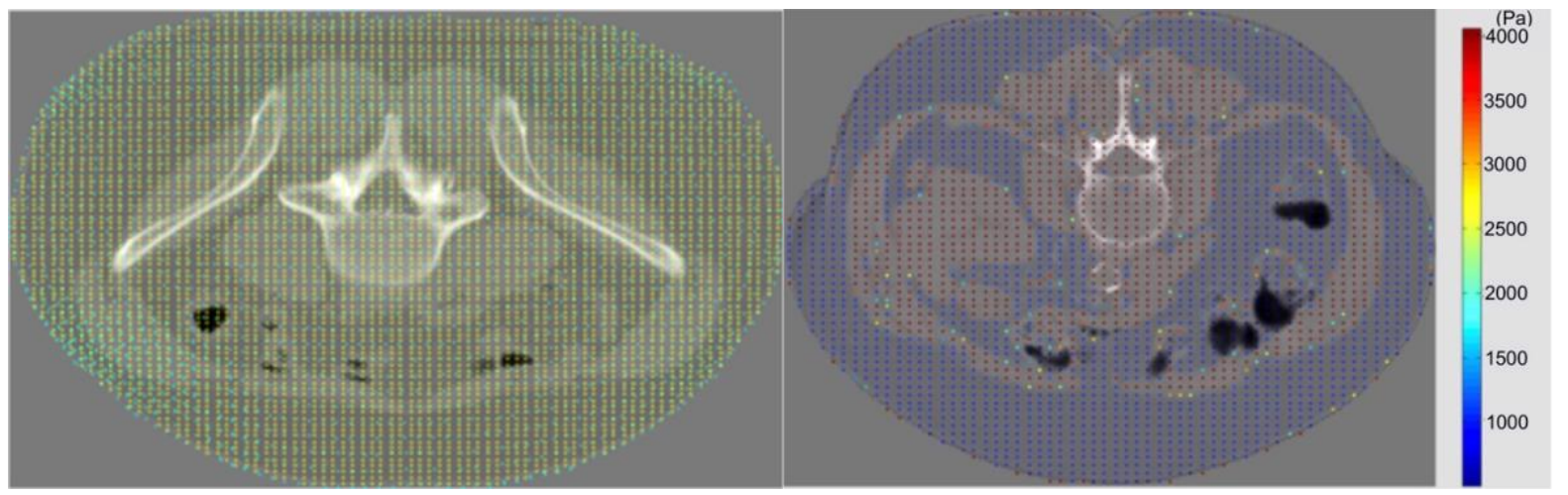

Figure 13 Example of meshless discretisation used by Li et al. (2016) when creating patient-specific computational biomechanics models. a) Distribution of support nodes and integration points on a selected transverse section. The blue crosses and yellow circles represent the support nodes and integration points, respectively. b) Assignment of the material properties using fuzzy tissue classification. The shear modulus magnitude is represented by a colour scale. Note that the integration points belonging to the same tissue type (indicated by the same colour) match the areas where the image intensity is similar. Only local tissue misclassification is present. This can be seen as a local variation in the integration point colour (where the adjacent integration points have different colour and, consequently, different shear modulus assigned) at the boundaries between different tissue types. Eight tissue types are distinguished: Type 1, 2 and 3 is for lungs and other gas-filled spaces (such as abdominal cavity), Type 4 - fat, Type 5 - muscles and abdominal organs, Type 6 - stomach and intestines, and Type 7 and 8 - bones (treated as rigid). Adapted from Li et al. (2016). 
Moving/Fixed Images

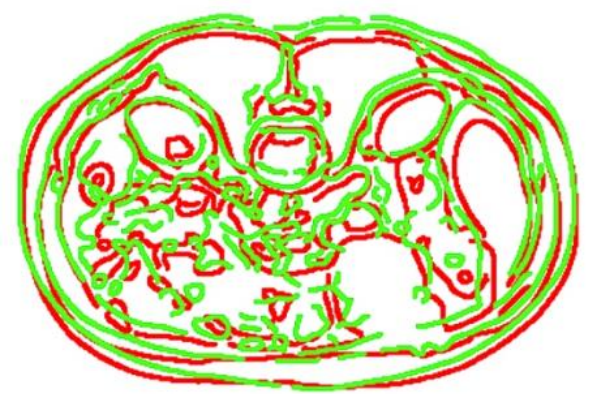

Case I
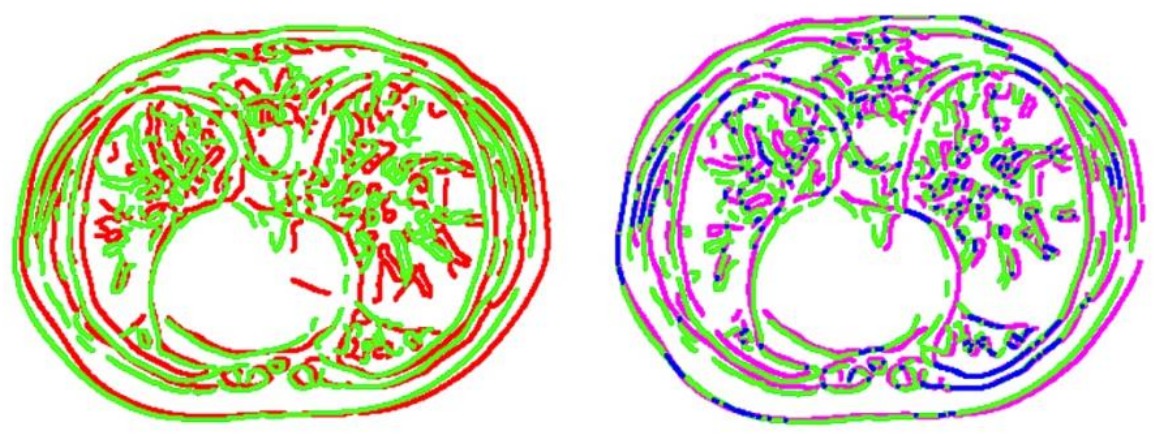

Case II
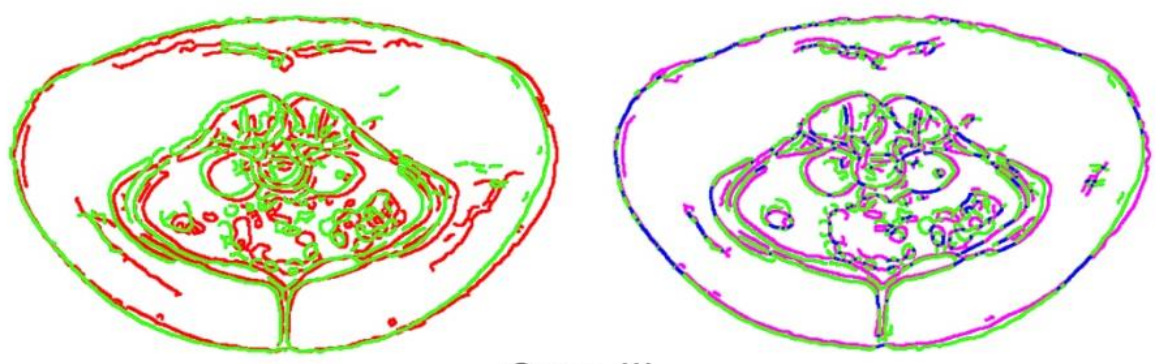

Case III

Figure 14 Results from Li et al. (2016): qualitative evaluation of biomechanics-based whole body CT image registration. The figure shows the Canny edges (detected using Canny edge filter (Canny, 1986)) in the moving, target (fixed) and registered (i.e. moving image warped using the deformation computed by biomechanical models) images. Left-hand-side column: comparison of the edges in the moving and fixed image. Right-hand-side column: comparison of the edges in the registered and target images. Edges in the moving image are indicated by red colour; edges in target image — by green; and the edges in the registered image — by pink. Good overlap (with some local misalignment) between the edges in registered and target images is evident. Adapted from Li et al. (2016). 


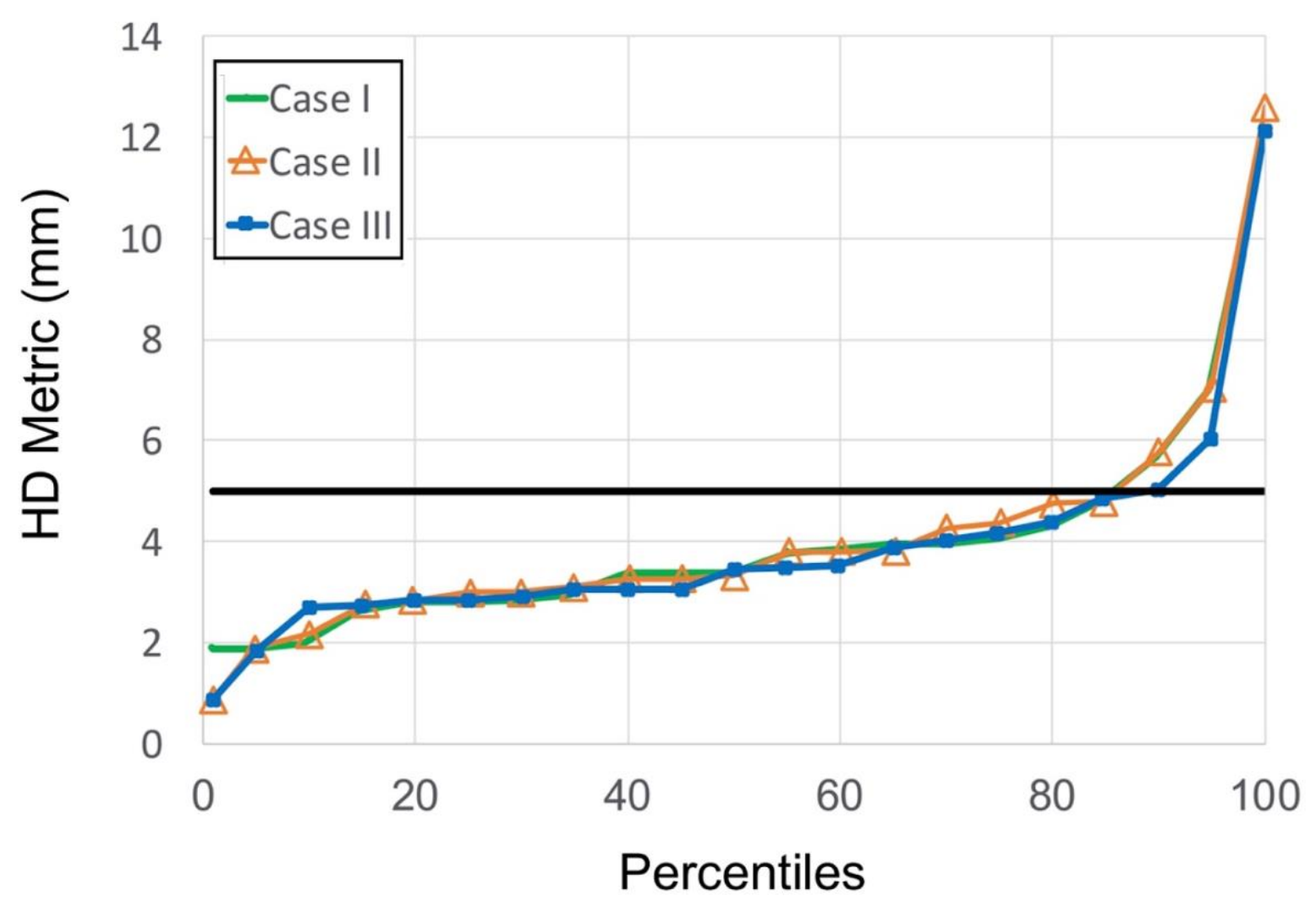

Figure 15 The percentile edge-based Hausdorff Distance (HD) metric for three cases/image-sets analysed in $\mathrm{Li}$ et al. (2016). Around $85 \%-95 \%$ of edges are accurately registered using the patient-specific non-linear meshless models created by Li et al. (2016). The percentile HD metric were measured in transverse sections for three image sets. The horizontal line is the $5.0 \mathrm{~mm}$ mark which is the two-times inplane resolution of the intra-operative images used in $\mathrm{Li}$ et al. (2016). For detailed discussion of application of the Hausdorff distance as an image similarity metric, see Garlapati el. (2015). Figure created from the results (figures) published in Li et al. (2016). 


\section{DISCUSSION}

The review and examples presented in this Chapter point to complementary roles of image analysis and computational biomechanics. Medical images are in practice the primary (if not the only) source of patient-specific information about the organ geometry and loading acting on the organs when creating computational biomechanics models for surgical simulation. Such models, on the other hand, can inform image analysis by computing deformation fields within organs during surgery. The computed deformations are then used to register (warp) high quality pre-operative images to the intra-operative organ geometry in a process known as biomechanicsbased image registration. Unlike image-based registration that relies solely on image processing techniques, the biomechanics-based registration using appropriate nonlinear formulation of computational mechanics, that accounts for both material (nonlinear stress-strain relationship) and geometric (large deformations of soft tissues) non-linearity, not only ensures plausibility of the predicted deformations but also does not require an intra-operative image. It should be noted that this requires also application of hyperelastic (or hyperviscoelastic) material models consistent with such formulation. Some authors choose linear elastic material model with geometrically non-linear solution procedure. This, however, may lead to erroneous results in the presence of large strain or element rotation (Bathe, 1996).

As shown in the examples discussed in sections Neuroimage registration, Magnetic Resonance (MR) image registration for intracranial electrode localisation for epilepsy treatment, and Whole body Computed Tomography (CT) image registration for image registration formulated as a Dirichlet-type problem of computational mechanics (where the loading is defined by prescribing deformations on the boundary of the analysed object), sparse information about deformations of the organ surface 
and neo-Hookean material model (the simplest hyperelastic model) are sufficient to facilitate biomechanics-based registration with the accuracy at least as high as that of the image-based registration using the intra-operative image. It is necessary to note that this conclusion is limited to intra-patient image registration as no examples of inter-patient registration were discussed in this Chapter.

Although formally many different methods can be used to solve the equations of non-linear continuum mechanics governing the behaviour of computational biomechanics models, for such models to be useful in a clinical workflow, patientspecific computational grids need to be generated in automated/semi-automated mode from medical images and robust and stable solution must be guaranteed without the requirement for the user to become an expert in computational mechanics. Traditionally used finite element method does not fulfil these conditions as it necessitates image segmentation to define closed surfaces for creation of patientspecific finite element meshes. Construction of such meshes is time consuming and requires involvement of an experienced analyst and the solution stability and accuracy deteriorate when the analysed organ undergoes large deformations and fragmentation (discontinuities) due to surgical dissection. Therefore, we recommend meshless methods of computational mechanics that utilise grids in a form of clouds of points (nodes). As shown in the example of biomechanics-based whole body image registration discussed in section Whole body Computed Tomography (CT) image registration such methods facilitate generation of patient-specific computational biomechanics models directly from medical image without any need for image segmentation. Recent developments in adaptive integration (Joldes et al., 2015) and shape functions (Chowdhury et al., 2017) for meshless discretisation reduce the requirements for expert knowledge in computational mechanics when creating 
meshless computational grids as the solution accuracy is guaranteed for almost arbitrary node placement.

\section{ACKNOWLEDGEMENTS}

The funding from the Australian Government through the Australian Research Council ARC (Discovery Project Grants DP160100714, DP1092893, and DP120100402) and National Health and Medical Research Council NHMRC (Project Grants APP1006031 and APP1144519) is gratefully acknowledged.

\section{REFERENCES}

Ateshian, G.A., Henak, C.R., Weiss, J.A., 2015. Toward patient-specific articular contact mechanics. Journal of Biomechanics 48, 779-786.

Baker, T.J., 1989. Automatic mesh generation for complex three-dimensional regions using a constrained delaunay triangulation. Eng. Comput. 5, 161-175.

Bathe, K.-J., 1996. Finite Element Procedures. Prentice-Hall, Upper Saddle River, ISBN 0-13-3014584.

Bauman, J.A., Feoli, E., Romanelli, P., Doyle, W.K., Devinsky, O., Weiner, H.L., 2008. Multistage epilepsy surgery: Safety, efficacy, and utility of a novel approach in pediatric extratemporal epilepsy. Neurosurgery 62, SHC-489-SHC-505.

Belwadi, A., Yang, K.H., 2011. Mechanism of aortic injury in nearside left lateral automotive crashes: A finite element accident injury reconstruction approach, in Proc. of 2011 IRCOBI Conference on the Biomechanics of Impact, International Research Council on the Biomechanics of Injury, Krakow; Poland; 14 September 2011 through 16 September 2011, pp. 169-180.

Belytschko, T., Liu, W.K., Moran, B., 2006. Nonlinear Finite Elements for Continua and Structures. John Wiley\&Sons, Chichester.

Belytschko, T., Rabczuk, T., Huerta, A., Fernández-Méndez, S., 2004. Meshfree Methods, in: E. Stein, Borst, R., Hughes, T.J. (Eds.), Encyclopedia of Computational Mechanics. Wiley, Chichester.

Beucher, S., Meyer, F., 1993. The morphological approach to segmentation: The watershed transformation, in: E. R. Dougherty (Ed.), Mathematical Morphology in Image Processing. Dekker, New York, pp. 433-482.

Bilston, L.E., 2011. Brain Tissue Mechanical Properties, in: Biomechanics of the Brain, Miller, K. (Ed.). Springer, pp. 69-89. 1st edition

Bilston, L. E., 2018: Brain Tissue Mechanical Properties, in: Biomechanics of the Brain, Miller, K. (Ed.). Springer, 2nd edition. Springer. Accepted.

Blacker, T.D., Meyers, R.J., 1993. Seams and wedges in plastering: A 3-D hexahedral mesh generation algorithm. Eng. Comput. 9, 83-93.

Bonet, J., Burton, A.J., 1998. A simple average nodal pressure tetrahedral element for incompressible and nearly incompressible dynamic explicit applications. Commun. Numer. Methods Eng. 14, 437-449. 
Bostrom, O., Svensson, M.Y., Aldman, B., Hansson, H.A., Haland, Y., Lovsund, P., Seeman, T., Suneson, A., Saljo, A., Ortengren, T., 1996. A new neck injury criterion candidade - based on injury findings in the cervical spinal ganglia after experimental neck extension trauma, in Proc. of 1996 International IRCOBI Conference On the Biomechanics of Impact. International Reserch Council On the Biomechanics of Injury, Dublin, Ireland, pp. 123-136.

Bottan, S., Poulikakos, D., Kurtcuoglu, V., 2012. Phantom model of physiologic intracranial pressure and cerebrospinal fluid dynamics. IEEE Transactions on Biomedical Engineering 59, 1532-1538.

Bucholz, R., MacNeil, W., McDurmont, L., 2004. The operating room of the future. Clinical Neurosurgery 51, 228-237.

Bucki, M., Lobos, C., Payan, Y., 2010. A fast and robust patient specific Finite Element mesh registration technique: Application to 60 clinical cases. Medical Image Analysis 14, 303-317.

Canny, J., 1986. A computational approach to edge detection. IEEE Transactions on Pattern Analysis and Machine Intelligence PAMI-8, 679-698.

Carey, G. F., 1997. Computational Grids: Generations, Adaptation \& Solution Strategies Taylor \& Francis.

Cavendish, J.C., Field, D.A., Frey, W.H., 1985. An approach to automatic 3-dimensional finite-element mesh generation. International Journal for Numerical Methods in Engineering 21, 329-347.

Chaudhuri, P.K., Low, B.C., Lim, C.T., 2018. Mechanobiology of Tumor Growth. Chemical Reviews 118, 6499-6515.

Choi, W.Y., Kwak, D.Y., Son, I.H., Im, Y.T., 2003. Tetrahedral mesh generation based on advancing front technique and optimization scheme. International Journal for Numerical Methods in Engineering $58,1857-1872$.

Chowdhury, H.A., Wittek, A., Miller, K., Joldes, G.R., 2017. An Element Free Galerkin Method Based on the Modified Moving Least Squares Approximation. J Sci Comput 71, 1197-1211.

Ciarlet, P.G., 1988. Mathematical Elasticity. North Hollad. The Netherlands.

Dassault Systèmes Simulia Corporation: ABAQUS Online Documentation (2014).

Davis, L.S., 1975. A survey of edge detection techniques. Computer Graphics and Image Processing 4, 248-270.

Doblare, M., Cueto, E., Calvo, B., Martinez, M.A., Garcia, J.M., Cegonino, J., 2005. On the employ of meshless methods in biomechanics. Computer Methods in Applied Mechanics and Engineering 194, 801-821.

Dutta-Roy, T., Wittek, A., Miller, K., 2008. Biomechanical modelling of normal pressure hydrocephalus. Journal of Biomechanics 41, 2263-2271.

Ertl, M., Raasch, N., Hammel, G., Harter, K., Lang, C., 2018. Transtemporal investigation of brain parenchyma elasticity using 2-D shear wave elastography: Definition of age-matched normal values. Ultrasound in Medicine \& Biology 44, 78-84.

Fan, X., Roberts, D.W., Schaewe, T.J., Ji, S., Holton, L.H., Simon, D.A., Paulsen, K.D., 2017. Intraoperative image updating for brain shift following dural opening. Journal of Neurosurgery 126, 1924-1933.

Fedorov, A., Beichel, R., Kalpathy-Cramer, J., Finet, J., Fillion-Robin, J.-C., Pujol, S., Bauer, C., Jennings, D., Fennessy, F., Sonka, M., Buatti, J., Aylward, S., Miller, J.V., Pieper, S., Kikinis, R., 2012. 3D Slicer as an image computing platform for the Quantitative Imaging Network. Magnetic Resonance Imaging 30, 1323-1341.

Fung, Y.C., 1993. Biomechanics.Mechanical Properties of Living Tissues, Second ed. Springer-Verlag, New York, pp. 392-426.

Garlapati, R.R., Roy, A., Joldes, G.R., Wittek, A., Mostayed, A., Doyle, B., Warfield, S.K., Kikinis, R., Knuckey, N., Bunt, S., Miller, K., 2014. More accurate neuronavigation data provided by biomechanical modeling instead of rigid registration. Journal of Neurosurgery 120, 1477-1483.

Garlapati, R.R., Mostayed, A., Joldes, G.R., Wittek, A., Doyle, B., Miller, K., 2015. Towards measuring neuroimage misalignment. Computers in Biology and Medicine 64, 12-23.

Geuzaine, C. and Renacle, J.-F., 2016. Fifth Workshop on Grid Generation for Numerical Computations. Liege, Belgium; http://tetrahedron.montefiore.ulg.ac.be/ 
Graham, S., Taylor, R., Vannier, M., 2000. Needs Assessment for Computer-Integrated Surgery Systems, in: Delp, S., DiGoia, A., Jaramaz, B. (Eds.), Medical Image Computing and ComputerAssisted Intervention MICCAI 2000. Lecture Notes in Computer Science 1935, pp. 931-939, Springer Berlin Heidelberg, ISBN 978-3-540-41189-5.

Grimson, W.E.L., Leventon, M.E., Ettinger, G., Chabrerie, A., Ozlen, F., Nakajima, S., Atsumi, H., Kikinis, R., Black, P., 1998. Clinical experience with a high precision image-guided neurosurgery system, Lecture Notes in Computer Science 1496, pp. 63-73.

Grosland, N.M., Shivanna, K.H., Magnotta, V.A., Kallemeyn, N.A., DeVries, N.A., Tadepalli, S.C., Lisle, C., 2009. IA-FEMesh: An open-source, interactive, multiblock approach to anatomic finite element model development. Computer Methods and Programs in Biomedicine 94, 96-107.

Guo, J., Hirsch, S., Fehlner, A., Papazoglou, S., Scheel, M., Braun, J., Sack, I., 2013. Towards an Elastographic Atlas of Brain Anatomy. PLOS ONE 8, e71807-1-10.

Hausdorff, F., 1957. Set Theory. Chelsea Publishing Company, New York.

Hiscox, L.V., Johnson, C.L., Barnhill, E., McGarry, M.D.J., Huston, J.r., van Beek, E.J.R., Starr, J.M., Roberts, N., 2016. Magnetic resonance elastography (MRE) of the human brain: technique, findings and clinical applications. Physics in Medicine \& Biology 61, R401-R437.

Ho-Le, K., 1988. Finite element mesh generation methods: a review and classification. Computer-Aided Design 20, 27-38.

Horton, A., Wittek, A., Joldes, G.R., Miller, K., 2010. A meshless Total Lagrangian explicit dynamics algorithm for surgical simulation. International Journal for Numerical Methods in Biomedical Engineering 26, 977-998.

Hu, K., Zhang, Y.J., 2016. Centroidal Voronoi tessellation based polycube construction for adaptive allhexahedral mesh generation. Computer Methods in Applied Mechanics and Engineering 305, 405-421.

Hughes, T.J.R., 2000. The Finite Element Method: Linear Static and Dynamic Finite Element Analysis. Dover Publications, Mineola.

Ito, Y., Shih, A.M., Soni, B.K., 2009. Octree-based reasonable-quality hexahedral mesh generation using a new set of refinement templates. International Journal for Numerical Methods in Engineering 77, 1809-1833.

Jiang, Y., Li, G., Qian, L.-X., Liang, S., Destrade, M., Cao, Y., 2015. Measuring the linear and nonlinear elastic properties of brain tissue with shear waves and inverse analysis. Biomechanics and Modeling in Mechanobiology 14, 1119-1128.

Jin, X., Joldes, G.R., Miller, K., Yang, K.H., Wittek, A., 2014. Meshless algorithm for soft tissue cutting in surgical simulation Computer Methods in Biomechanics and Biomedical Engineering 17, 800-817.

Joldes, G.R., Wittek, A., Miller, K., 2009a. Non-locking tetrahedral finite element for surgical simulation. Commun. Numer. Methods Eng. 25, 827-836.

Joldes, G.R., Wittek, A., Miller, K., 2009b. Suite of finite element algorithms for accurate computation of soft tissue deformation for surgical simulation. Medical Image Analysis 13, 912-919.

Joldes, G.R., Chowdhury, H., Wittek, A., Miller, K., 2017. A new method for essential boundary conditions imposition in explicit meshless methods. Engineering Analysis with Boundary Elements 80, 94-104.

Joldes, G.R., Wittek, A., Miller, K., 2010. Real-time nonlinear finite element computations on GPU Application to neurosurgical simulation. Computer Methods in Applied Mechanics and Engineering 199, 3305-3314.

Joldes, G.R., Wittek, A., Miller, K., 2012. Stable time step estimates for mesh-free particle methods. International Journal for Numerical Methods in Engineering 91, 450-456.

Joldes, G.R., Wittek, A., Miller, K., 2015. Adaptive numerical integration in Element-Free Galerkin methods for elliptic boundary value problems. Engineering Analysis with Boundary Elements 51, 52-63.

Kapur, T., Grimson, W.E.L., Wells, W.M., Kikinis, R., 1996. Segmentation of brain tissue from magnetic resonance images. Medical Image Analysis 1, 109-127.

Kikuchi, T., Togawa, A., Murakami, D., Tatsu, K., Pal, C., Okabe, T., 2015. A study of brain injury mechanisms in vehicle crashes, 24th Enhanced Safety of Vehicles ESV Conference, Gothenburg, Sweden. 
Li, M., Miller, K., Joldes, G.R., Doyle, B., Garlapati, R.R., Kikinis, R., Wittek, A., 2015. Patient-specific biomechanical model as whole-body CT image registration tool. Medical Image Analysis 22, 22-34.

Li, M., Miller, K., Joldes, G.R., Kikinis, R., Wittek, A., 2016. Biomechanical model for computing deformations for whole-body image registration: A meshless approach. International Journal for Numerical Methods in Biomedical Engineering 32, e02771-02771-02718

Li, S. and Liu, W.K., 2004. Meshfree Particle Methods. Springer-Verlag, Berlin Heidleberg, ISBN 9783-540-22256-9.

Liu, G.R., 2003. Mesh Free Methods: Moving Beyond the Finite Element Method. CRC Press, Boca Raton.

Liow, Y.-T., 1991. A contour tracing algorithm that preserves common boundaries between regions. CVGIP: Image Understanding 53, 313-321.

Lohner, R., 1996. Progress in grid generation via the advancing front technique. Eng. Comput. 12, 186210.

Marechal, L., 2009. Advances in Octree-Based All-Hexahedral Mesh Generation: Handling Sharp Features, in: Clark, B. (Ed.), Proc. of the 18th International Meshing Roundtable. Springer Berlin Heidelberg, pp. 65-84.

Margulies, S.S., Thibault, L.E., 1989. An analytical model of traumatic diffuse brain injury. Journal of Biomechanical Engineering - Transactions of the ASME 111, 241-249.

Matsushita, T., Yamazaki, N., Sato, T., Hirabayashi, K., 1997. Biomechanical and medical investigations of human neck injury in low velocity collisions. Neuro-Orthopaedics 21, 27-45.

Menage, L.P.M., 2017. "Computer Simulation of Brain Deformations for the Surgical Treatment of Paediatric Epilepsy", School of Mechanical and Chemical Engineering. MSc (Master of Professional Engineering) Thesis, The University of Western Australia, Perth, Western Australia, Australia.

Miller, K., Horton, A., Joldes, G.R., Wittek, A., 2012. Beyond finite elements: A comprehensive, patientspecific neurosurgical simulation utilizing a meshless method. Journal of Biomechanics 45, 2698 - 2701.

Miller, K., Joldes, G., Lance, D., Wittek, A., 2007. Total Lagrangian explicit dynamics finite element algorithm for computing soft tissue deformation. Commun. Numer. Methods Eng. 23, 121-134.

Miller, K., Joldes, G.R., Bourantas, G.C., Warfield, S.K., Hyde, D.E., Kikinis, R., Wittek, A., 2019. Biomechanical modeling and computer simulation of the brain during neurosurgery, Medical Image Analysis, e3250-1-24, https://onlinelibrary.wiley.com/doi/epdf/10.1002/cnm.3250.

Miller, K., Lu, J., 2013. On the prospect of patient-specific biomechanics without patient-specific properties of tissues. J Mech Behav Biomed Mater 27, 154-166.

Miller, K., Wittek, A., Joldes, G., 2011. Biomechanical modeling of the brain for computer-assisted neurosurgery, Biomechanics of the Brain. Springer New York, pp. 111-136.

Mostayed, A., Garlapati, R., Joldes, G., Wittek, A., Roy, A., Kikinis, R., Warfield, S., Miller, K., 2013. Biomechanical model as a registration tool for image-guided neurosurgery: Evaluation against BSpline registration. Ann Biomed Eng 41, 2409-2425.

Ono, K., Kaneoka, K., Wittek, A., Kajzer, J., 1997. Cervical injury mechanism based on the analysis of human cervical vertebral motion and head-neck-torso kinematics during low speed rear impacts, in Proc. of 41st Stapp Car Crash Conference. Society of Automotive Engineers, Inc., Lake Buena Vista, Florida, USA, pp. 339-356.

Otsu, N., 1979. A Threshold selection method from gray-level histograms. IEEE Transactions on Systems, Man, and Cybernetics 9, 62-66.

Panjabi, M.M., Ito, S., Ivancic, P.C., Rubin, W., 2005. Evaluation of the intervertebral neck injury criterion using simulated rear impacts. Journal of Biomechanics 38, 1694-1701.

Perotto, S. and Formaggia, L., 2013. New Challenges in Grid Generation and Adaptivity for Scientific Computing. Fourth Tetrahedron Workshop on Grid Generation for Numerical Computations. SEMA SIMAI Springer Series. Springer.ISBN: 978-3-319-35926-7; doi: 10.1007/978-3-319-06053-8

Pohl, K.M., Bouix, S., Nakamura, M., Rohlfing, T., McCarley, R.W., Kikinis, R., Grimson, W.E.L., Shenton, M.E., Wells, W.M., 2007. A hierarchical algorithm for MR brain image parcellation. IEEE Transactions on Medical Imaging 26, 1201-1212. 
Psanoudakis, N., 2017. "Computer Simulation of Brain Deformation for Paediatric Epilepsy", MSc Thesis, Department of Mechanical Engineering, The University of Western Australia,, Perth-Crawley, Western Australia, Australia.

Ramme, A.J., Criswell, A.J., Wolf, B.R., Magnotta, V.A., Grosland, N.M., 2011. EM Segmentation of the Distal Femur and Proximal Tibia: A High-Throughput Approach to Anatomic Surface Generation. Ann Biomed Eng 39, 1555-1562.

Rohan, P.Y., Lobos, C., Nazari, M.A., Perrier, P., Payan, Y., 2014. Finite element modelling of nearly incompressible materials and volumetric locking: a case study. Computer Methods in Biomechanics and Biomedical Engineering 17, 192-193.

Sethian, J.A., 1999. Set Methods and Fast Marching Methods: Evolving Interfaces in Computational Geometry, Fluid Mechanics, Computer Vision and Materials Science. Cambridge University Press. ISBN-13: 9780521645577.

Shewchuk, J.R., 2002. Delaunay refinement algorithms for triangular mesh generation. Computational Geometry 22, 21-74.

Takhounts, E.G., Craig, M.J., Moorhouse, K., McFadden, J., Hasija, V., 2013. Development of Brain Injury Criteria (BrlC). Stapp Crash Crash Journal 57, 243-266.

Tautges, T.J., Blacker, T., Mitchell, S.A., 1996. The whisker weaving algorithm: A connectivity-based method for constructing all-hexahedralfinite element meshes. S. International Journal for Numerical Methods in Engineering 39, 3327-3349.

Vemaganti, K., Joldes, G.R., Miller, K., Wittek, A., 2011. Total lagrangian explicit dynamics-based simulation of tissue tearing, in Wittek, A., Nielsen, P. M. F. and Miller, K (Eds.) Computational Biomechanics for Medicine: Soft tissues and Musculoskeletal Systems, Springer, New York, NY, pp. 63-72, ISBN 978-1-4419-9618-3.

Wittek, A., Grosland, N., Joldes, G., Magnotta, V., Miller, K., 2016. From Finite Element Meshes to Clouds of Points: A Review of Methods for Generation of Computational Biomechanics Models for Patient-Specific Applications. Ann Biomed Eng 44, 3-15.

Wittek, A., Hawkins, T., Miller, K., 2009. On the unimportance of constitutive models in computing brain deformation for image-guided surgery. Biomechanics and Modeling in Mechanobiology 8, 77-84.

Wittek, A., Joldes, G., Couton, M., Warfield, S.K., Miller, K., 2010. Patient-specific non-linear finite element modelling for predicting soft organ deformation in real-time; Application to non-rigid neuroimage registration. Progress in Biophysics and Molecular Biology 103, 292 - 303.

Wittek, A., Joldes, G., Miller, K., 2011. Algorithms for Computational Biomechanics of the Brain, in: Miller, K. (Ed.), Biomechanics of the Brain, pp. 189-219.

Wittek, A., Miller, K., Kikinis, R., Warfield, S.K., 2007. Patient-specific model of brain deformation: Application to medical image registration. Journal of Biomechanics 40, 919-929.

Yang, K., King, A., 2011. Modeling of the Brain for Injury Simulation and Prevention, in: Miller, K. (Ed.), Biomechanics of the Brain. Springer New York, pp. 91-110.

Yang, K.H., Shen, K.L., Demetropoulos, C.K., King, A.I., Kolodziej, P., Levine, R.S., Fitzgerald, R.H., 1996. The relationship between loading conditions and fracture patterns of the proximal femur. Journal of Biomechanical Engineering-Transactions of the ASME 118, 575-578.

Zhang, G.Y., Wittek, A., Joldes, G.R., Jin, X., Miller, K., 2014. A three-dimensional nonlinear meshfree algorithm for simulating mechanical responses of soft tissue. Engineering Analysis with Boundary Elements 42, 60-66.

Zhang, J.Y., Joldes, G.R., Wittek, A., Miller, K., 2012. Patient-specific computational biomechanics of the brain without segmentation and meshing. International Journal for Numerical Methods in Biomedical Engineering 29, 293-308.

Zhang, Y., Bajaj, C., 2006. Adaptive and quality quadrilateral/hexahedral meshing from volumetric data. Computer Methods in Applied Mechanics and Engineering 195, 942-960.

Zhang, Y., Hughes, T.J.R., Bajaj, C.L., 2010. An automatic 3D mesh generation method for domains with multiple materials. Computer Methods in Applied Mechanics and Engineering 199, 405-415. 